\title{
Community psychiatry and the National Institute of Mental Health
}

The modern Western evolution of Community Mental Health began as a "consumer led" movement in the United States in the 1930s. The U.S. Public Health Service planned at that time the National Institute of Mental Health, whose setting up by Federal legislation (the National Mental Health Act of 1946) was delayed by the Second World War. Important developments during the 1950s including the appearance of powerful psychoactive medications and improved techniques in epidemiology, were of further great importance. In Ireland the Mental Health legislation of 1945 with its subsequent amendments was accompanied by various far-seeing reports on the topic of mental health developments including community influences. The influence of the National Institute of Mental Health cannot be underestimated in terms of its impact in both theoretical and applied mental health research. The developments in the United States which have subsequently spread to Western Europe and other countries have only been possible through scientific application of research, for the most part fostered and funded by NIMH.

Community psychiatry is a widely misunderstood concept. Many seem to believe that community psychiatry refers only to the locus where patients are treated. Of course, community psychiatry is a developing body of scientific information and practice using psychiatric and social principles to bring services to large population groups.

A major step forward in Ireland was the publication of the Government's policy "Planning for the Future",' suggesting a number of areas for development which, of course, require further research into efficacy and application in an Irish context.

To practice medicine without this body of psychiatric research would be like flying an aircraft without instruments. Both practices are likely to lead to, at the minimum, waste of scarce resources and, at the worst, tragedy on a large scale.

Funding for mental health research at the best of times is fraught with difficulty and scepticism. The NIMH was set up as a branch of the National Institutes of Health in order to foster the Cinderella science of psychiatry for the better good of American patients. Perhaps the time has now come to set up a similar National Institute of Mental Health in Ireland to aid education, to ensure that research principles are applied to testing new assertions, and to help in the monitoring of the implementation of policy such as Planning for the Future. ${ }^{1}$ Already the Irish Psychiatric Training Committee has expressed the hope that a special body should be set up to foster health research in the area of mental health needs of the country. In times of scarcity it is all the more desirable to protect important areas, misconceived by many as being of low priority.

Prof. R J Daly

University College Cork, Professorial Psychiatric Unit, Cork Regional Haspital.

\section{Reference}

1. The psychiatric service - planning for the future. Report of a study group on the development of psychiatric services. Dublin: Publ Stationery Office, 1984.

\section{An Irish National Institute of Mental Health}

Professor Daly has called for the formation of a National Institute of Mental Health of Ireland. This idea has been mooted in various forms. But the most popular organisation currently engaged in the monitoring of standards of training and care - the Royal College of Psychiatrists (and with it the Irish Psychiatric Training Committee) - has the support of the vast majority of psychiatrists in this country. However, it does not provide funding for psychiatric research in Ireland.

Initially at least, a new National Institute of Mental Health of Ireland should be conceived primarily neither as an educational nor a monitoring body, nor a site where research is planned or takes place. Rather it should confine itself single mindedly to the acquisition and disbursement of money, in support of worthwhile research whose protocols have been submitted to it. A few colleagues amongst us with entrepreneurial skills have shown that it is possible, mainly bypassing the Irish government and semi-state bodies, to attract large sums of money for research.

An Irish National Institute of Mental Health must be, and be seen to be, a broadly based body, not the creature of any person or clique, not even the Irish Division of the Royal College of Psychiatrists. It must be supported and have representatives not only from the medical schools, but from researchers outside them; from both public and private sectors; from Consultants and trainees; from several disciplines; and from Ireland and Northern Ireland.

Whatever factors may hinder psychiatric research in Ireland, insufficient funding need not be one of them. Two or three activists, with the broad based support of their colleagues' good will, could create the answer to this wish and need.

Mark Hartman, Editor 